\title{
Impact of Narrative Exposure Therapy on torture survivors in the MENA region
}

\author{
Ane Kirstine Viller Hansen MSc.*, Nete Sloth Hansen-Nord MSc*, Issam Smeir EdD*, \\ Lianne Engelkes-Heby BSc*, Jens Modvig MD, PhD* \\ Contributors who collected data for this research: Malek Lakhoua ${ }^{1}$, Anissa Bouaske ${ }^{1}$, Linda Dridi ${ }^{1}$, Ameera Abdul \\ Kareem Marran Marie ${ }^{2}$, Mohammed Azeez Raheem ${ }^{3}$, Chro Qadir ${ }^{3}$, Hani Mostafa ${ }^{4}$, Raghda Sleit ${ }^{4}$, Hussein \\ Alsalem 5 , Mohammad Qatawneh ${ }^{5}$, Areej Sumreen ${ }^{5}$, Sana Hamzeh ${ }^{6}$, Marie Abdel Ahad ${ }^{6}$, Rahil Bahij ${ }^{6}$, Nancy \\ Jabbour ${ }^{6}$, Fatima Alqaadi ${ }^{7}$, Manar Arar ${ }^{8}$, Raya Farsakh ${ }^{8}$, Sanaa Mohammad $^{9}$
}

\section{Key points of interest:}

- Narrative Exposure Therapy treatment of survivors of torture and war living in unsafe and difficult environments in the Middle East and North Africa region was associated with a reduction in symptoms of post-traumatic stress disorder, anxiety and depression, pain perceptions and levels of disability, and improvements in self-reported health.

- This research suggests a positive influence of minimizing language barriers between therapist and client, and staying in a familiar cultural context while receiving Narrative Exposure Therapy.

- This research suggests that trauma treatment should not be delayed until refugees or asylum seekers resettle to live in a stable and permanent environment.

^) DIGNITY - Danish Institute Against Torture, Denmark

Correspondence to: nsh@dignityinstitute.dk

1) Tunisian Institute of Rehabilitation of Torture Survivors (Nebras), Tunisia

2) Bahjat Al-Fuad rehabilitation centre for torture victims, Iraq

\begin{abstract}
Introduction: Narrative Exposure Therapy (NET) is a brief cognitive-behavioural intervention for individuals with posttraumatic stress disorder (PTSD) which has mostly been used to treat traumatised asylum seekers and refugees in highincome settings. Evidence is scarce on the effectiveness of NET with torture survivors, especially in the Middle East and North African (MENA) region where health systems are unable to meet the increasing needs of mental health disorders caused by war and displacement. Methods: During the period 2013 to 2016 DIGNITY - Danish Institute Against Torture, in collaboration with partners, implemented a capacity-building training programme on NET among 44 Arabic health professionals from highly specialised torture rehabilitation centers in Jordan, Palestine, Egypt, Lebanon,
\end{abstract}

Wchan Organisation for Victims of Human Rights Violations, Iraq

4) $\mathrm{Al}$ Nadeem Centre for Torture Victims, Egypt

$\left.{ }^{5}\right)$ Nour al Hussein Foundation - Institute for Family Health, Jordan

${ }^{6}$ ) Restart Center for Rehabilitation of Victims of Violence and Torture, Lebanon

${ }^{7}$ ) Al Fanar Rehabilitation Center for Victims of Violence and Torture, Sudan

8) Treatment and Rehabilitation Center for Victims of Torture (TRC), Palestine

$\left.{ }^{9}\right)$ Syrian Bright Future, Syria 
Iraq, Tunisia, Libya, Sudan and Syria. A multi-centre study was carried out across all centres comprising of the collection of client data on socio-demographic variables, torture exposure, and psychosomatic health indicators. Clinical assessment of mental health symptoms among torture survivors was performed by the NET therapists pre- and post NET therapy and at four months' follow-up, and means were compared. Results: Our findings show a statistically significant reduction in average psychological symptom load for PTSD (from 3.20 to 1.80 ), anxiety (2.78 - 1.61) and depression (2.75 - 1.96) with the largest effect on PTSD symptoms, and a larger effect for women than men. The results indicate improvements in selfreported health (3.85-2.82) and physical disabilities (2.90-1.76), as well as reduction in pain perceptions after therapy (4.44 -3.44). The duration of treatment was three months on average with a span from one to eight months. Discussion: This study provides new evidence suggesting a strong positive effect of NET in an Arab cultural setting which remains under-represented in the NET evidence base. However, some important limitations of the study preclude drawing firm conclusions, namely the lack of a control group, a high number of dropouts in follow up data and a potential risk of information bias. Contexts familiar to the torture survivor and shared cultural norms and language between the client and the therapist might positively affect the effect of NET on PTSD symptoms. This capacity-building training programme established a community of Arab trauma mental health experts in the MENA-region, and their implementation of NET was associated in time with a reduction of the mental health symptom load of survivors of torture and war.
Keywords: Torture, Middle East and North Africa, Narrative Exposure Therapy, rehabilitation, PTSD

\section{Introduction}

The burden of disease caused by armed conflict and violence is rising (Robjant \& Fazel, 2010). In 2011, when the Arab spring was initiated, people of the Middle East and North Africa (MENA) region were attempting to end corruption, increase political participation, rebel against oppression, and bring greater economic opportunities to their homelands. However, the Arab Spring led to the displacement of millions of refugees and asylum seekers as the region was consumed by civil wars, and widespread human right violations, including torture, increased. Alongside this rise, the mode of armed conflict and violence changed, affecting health-related outcomes and approaches.

Torture survivors and refugees experience complex trauma, including sustained exposure to repeated or multiple traumatic incidents, often of an interpersonal nature, occurring in circumstances where escape is impossible (Mørkved, 2014). Refugees are vulnerable to developing mental health disorders including post-traumatic stress disorder (PTSD) (Gwozdziewycz \& Mehl-Madrona, 2013). Asylum-seekers, who often have experienced the same pre-displacement stressors as refugees, are even more vulnerable to PTSD due to their insecure residential status (Schauer, Neuner, \& Elbert, 2005; Crumlish \& O'Rourke, 2010). Miller and Rasmussen (2010) state that daily stressors influence the relationship between armed conflict and mental health, emphasizing the role of daily stressors on mental health among people exposed to war. These daily stressors include language barriers, cultural differences, 
instability, low social status, low employment rates, poor living conditions and lack of social support which might be related to having refugee status in new countries (Budosan, Aziz, Benner, \& Abras, 2016).

To successfully treat PTSD, the psychological and biological processes which form the basis for the condition must be addressed. Efforts have been made to explain the three main symptoms of PTSD; re-experiencing of intrusive memories, active avoidance, and hyper-arousal. The patients are often unable to reconstruct the traumatic event in a coherent and chronological manner. This fragmented memory lacks contextual information, hence causing a sense of constant threat as the traumatic event is indistinguishable from the present context. Furthermore, the re-experiencing of intrusive memories occurs due to the way memories of traumatic events are activated by one or more internal or external cues (Brewin \& Holmes, 2003; Robjant \& Fazel, 2010).

Narrative Exposure Therapy (NET) is a manualised short-term cognitive-behavioural intervention targeting survivors of conflict and organised violence. NET was developed to treat clinical issues specifically observed in refugees who experienced repeated traumatisation. To meet the needs of this target group NET is delivered as a shortterm standardised therapy in emergency settings where there is scarce access to human and monetary resources (Schauer et al., 2005). This is essential since there is a documented shortage of mental health professionals in the MENA region. Global health observatory data shows that Jordan in 2014 possessed 0.51 psychiatrists and 0.27 psychologists per 100,000 residents (WHO, 2014). NET builds on components from other therapeutic approaches such as prolonged exposure therapy, trauma focused cognitive behaviour therapy (TFCBT), and testimonial therapy (TT) (Robjant \& Fazel 2010). During NET the patient is encouraged to create a narrative of the entire lifespan from birth to the present with a special focus on reporting all traumatic experiences. In this way, the patient narrates all stressful life events in chronological order (Schauer et al., 2005), which makes NET suitable for persons exposed to multiple traumatic events (Hensel-Dittmann et al., 2011). During the treatment sessions, the patient and the therapist collaborate on constructing a detailed narration of the patient's biography. The aim of the therapy is to create a chronological structure of the fragmented memories of the traumatic events. The narration should include the sensory, emotional and cognitive experiences of the patient. The patient's testimony is written down by the therapist after the end of each session. In the last session, the patient and the therapist sign the written testimony. The testimony can later serve as an eye-witness report documenting human rights violations. NET is a standardised approach and can be delivered in a relatively small number of sessions. Each session lasts between 60-120 minutes and is preferably organised with one or more sessions per week with a maximum of 14 days between each session (Robjant \& Fazel, 2010). Many refugees migrate frequently due to lack of employment or fear of deportation by authorities in refugee-hosting countries. Therefore, a brief treatment model minimises therapy having to end prematurely. It is argued that NET is applicable in many cultures, since it is based on an oral narrative tradition which is a common element in many cultures (Schauer et al., 2005) including many of those represented in the MENA region. Oral cultures utilise a form of 
communication in which knowledge, art, ideas and cultural norms are received, preserved, and transmitted orally from one generation to another mainly through stories or narration (Zaharna, 1995). Additionally, it is imperative for the listener to play an active role in communication. They must construct meaning from what they have heard, as the meaning is not always blatantly apparent. It is well-documented that cultural sensitivity has a positive impact on treatment outcomes (Whaley \& Davis, 2007), which makes NET an applicable therapeutic modality in the MENA region.

NET remains one of the best supported psychosocial interventions to effectively decrease PTSD symptoms, documented in seven randomized controlled trials (RCTs) among adults (Adenauer, et al., 2011; Hijazi et al., 2014; Morath et al., 2014; Neuner, Schauer, Klaschik, Karunakara, \& Elbert, 2004; Neuner et al., 2008; Neuner et al., 2010; Stenmark, Catani, Neuner, Elbert \& Holen, 2013). The results of the RCTs show that NET was effective in decreasing PTSD and depression symptoms relative to inactive controls among refugees and asylum seekers displaced in high-income countries (Nosé et al., 2017). Four of these studies included torture survivors among the clients treated of which two were from the Middle East (Adenauer et al., 2011; Hijazi et al., 2014). This study investigates the effect of NET on PTSD, anxiety and depression symptoms, as well as on disability, self-rated health and pain among torture survivors in the MENA region using a pre-post design.

\section{Methods}

The intervention: Based on the extent of the problem of torture survivors and traumatised refugees in the MENA region in combination with limited rehabilitation services available for this target group,
DIGNITY, in collaboration with MENA partners, decided to work to enhance torture survivors' and traumatised refugees' access to effective rehabilitation and justice in the MENA region through a Regional Rehabilitation project (2012-2017). The project developed a "NET capacity-building training model" which involved training and supervision of mental health professionals from rehabilitation centres in the MENA region. The aim was to empower local mental health professionals to become trainers and supervisors of NET so that they could disseminate their knowledge to colleagues and institutions in the region. The NET capacity-building training model allowed trainees to advance through three stages: practitioner, supervisor, and trainer. In the first stage, 'practitioner' trainees received a five-day training course on trauma theories, screening, diagnosis, and treatment utilising the NET approach. Trainees were then asked to treat three clinical cases using NET with online clinical supervision through Skype by NET experts. Trainees who successfully treated three cases using NET under supervision, and who showed interest in becoming supervisors, could advance to the second stage. In the second stage supervisor trainees were required to attend another five-day clinical supervision course based on the Discrimination Model of Supervision (Bernard \& Goodyear, 2009). In this model, the clinical supervisors play three roles throughout the supervision process: teacher, counselor and consultant. The Discrimination Model also highlights three areas of focus for building skills: intervention, conceptualisation and personalisation issues. Interventions examine how technical aspects of the therapeutic process are handled. Conceptualisation examines how trainees formulate case from theory, and finally personalisation issues 
focus on helping them to be aware of their own issues and how those impact their ability to counsel their clients effectively. Following completion of the course, trainees were matched with colleagues from subsequent groups of trainees and became their respective supervisors. The match was based on geographical location and, where necessary, the supervision sessions were conducted through Skype. Finally, those who completed five to seven clinical cases under supervision were trained to become supervisors. The third stage of becoming a trainer required attendance at a five-day Training of Trainers (ToT) course. All trainings were offered in Arabic by Arabic-speaking trainers and based on an Arabic ToT manual and a monitoring and evaluation manual. Trainees were educated in presenting and using the training materials. For the first time, training materials on NET were developed in Arabic, which ensured accessibility of training materials and quality of future trainings. The NET capacity-building training model contributed to the execution of NET therapy in each home country of the trained therapists. Under this project 44 psychologists and psychiatrists from Jordan, Palestine, Egypt, Lebanon, Iraq, Tunisia, Libya, Sudan and Syria were trained and supervised in NET, out of which 16 went on to become trainers of NET in their native countries. All mental health professionals collected clinical data on their NET clients. Because of the ToT approach the mental health professionals were not trained simultaneously and therefore only 19 distributed their data to the authors for analysis, documentation and publication.

Data collection: Selection criteria for receiving treatment was exposure to war trauma and/or torture and being above the age of
15 years, which at the same represented inclusion criteria for participation in the study. Clients who dropped out during the course of the treatment were excluded from the statistical analyses. A contextualised structured questionnaire to monitor the effect of NET was designed. The questionnaire consisted of various variables assessing socio-demographic background information, traumatic head injury, family support, torture history, self-rated health and pain. The questionnaire also comprised the Arabic version of the validated scales Harvard Trauma questionnaire (HTQ 1-16) (Shoeb, Weinstein \& Mollica, 2007) measuring PTSD, Hopkins symptoms checklist (HSCL-25) (Mahfoud et al., 2013) for anxiety and depression and WHO Disability Assessment Schedule 12 items (WHODAS 2.0) assessing disability and functioning, using official Arabic versions.

These questions in HTQ 1-16 provide a score derived from the first 16 items of the test which relate directly to the symptoms of PTSD as listed in the Diagnostic and Statistical Manual of Mental Disorders, ( $4^{\text {th }}$ Edition Revised (DSM-IVR)) with a cut-off value at 2.5 (average scores equal to or higher than 2.5 are considered indicative of PTSD) (Mollica et al., 1992).

Symptoms of anxiety and depression were assessed by the HSCL-25 with a cutoff value at 1.75 for depression and anxiety. We constructed dichotomous variables for the scores of PTSD, anxiety and depression. Self-rated health was examined with the single-item measure: "In general, would you say that your health is excellent, very good, good, fair, or poor?" on a five-point scale where 1 equals excellent and 5 equals poor). Self-rated health was included in the monitoring of NET, because it has been established as a strong predictor of mortality and morbidity (Idler \& Benyamini, 1997; 
Schnittker \& Bacak, 2014). The single item question "How much pain have you had during the last 4 weeks?" was used to assess pain on a 6-point scale where 0 reflected 'none' and 6 equalled 'very severe pain'. Health and disability were assessed using the 12-item WHO Disability Assessment Schedule (WHODAS 2.0). The WHODAS 2.0 assesses the level of functioning in six domains in life: cognition (understanding and communicating); mobility; self-care; getting along with others; life activities; and participation in society. For each item, respondents rate the degree to which they have experienced difficulty in that area of functioning over the past 30 days on a 5 -point scale $(1=$ None; $5=$ Extreme or Cannot Do). The WHODAS 2.0 provides an overall disability scale, where a higher score indicates greater disability. There are no widely agreed clinical cut-offs for the scale (Konecky B et al., 2014). However, normative data is defined in the WHODAS manual (WHO, 2010). The normal distribution is based on a multi-country Survey Study on Health and Responsiveness (WHO, 2000-2001) carried out in 61 countries worldwide. In this survey, it was intended to produce comparable population estimates across cultures and groups, all samples were selected from nationally representative sampling frames (Üstün TB et al. 2003). Exposure to torture was examined with a dichotomised variable saying, "Have you ever been exposed to torture?" The trainees were trained in identifying victims of torture from the definition set out in the United Nations Convention Against Torture and Cruel, Inhuman or Degrading Treatment. Furthermore, the clients were referred by UN organisations working with refugees or through local medical institutions.

The clients' symptoms of PTSD, anxiety and depression were assessed before therapy and at the final therapy session, as well as levels of pain, disability and selfrated health. Follow-up was intended to be conducted after four months and consisted of variables measuring symptoms of PTSD, anxiety and depression. A qualitative data component was developed to record the clients' perception of the course of treatment: "How has the treatment affected you in terms of how you feel, what you are able to do and how you think of all aspects of your life, e.g. your health and well-being, social relations and integration within the larger social system?". The qualitative data was collected by the therapists and written as notes by the therapists in a text box in the questionnaire. Before receiving therapy, the clients were informed about the purpose of the data collection and that the study protects anonymity. Informed consent was given orally by clients to the therapists and registered in writing by the therapists. Clients were informed that they had the right to withdraw from the therapy at any time for any reason. Ethical approval was obtained from an internal ethical committee which follows the ethical guidelines of the European Research Council (ERC). The rehabilitation centres managed all data in compliance with protocols for the protection of human subjects in their respective countries, where they existed.

Statistical analyses: Health status (symptoms of PTSD, anxiety and depression, selfreported health, pain and disability) was compared before and after therapy. Comparisons of the mean score for PTSD, anxiety, depression, pain and disability were performed using paired-samples t-test. The assumption of homogeneity of variances was tested using Levene's test. Self-rated health was tested by Pearsons $\chi 2$-test. The level of disability was plotted into a diagram 
before and after therapy and compared to the distribution score in a normal population defined in the WHODAS manual (WHO, 2010). Furthermore, the mean score was calculated pre- and post-therapy and compared to percentiles for the said normal population. The effect of the therapy was further evaluated by comparisons of the qualitative data and the quantitative data. All analyses were conducted with a two-sided level of significance $(\mathrm{p}<0.05)$ and calculated in SPSS 19.0.

\section{Results}

Table 1 shows the distribution of socioeconomic baseline data regarding 110 torture survivors and traumatised refugees who received NET. The proportion of men was higher than women, and the majority were in the age range 36-45 years. The level of education was almost equally distributed, with most people having completed primary school. The majority of clients were Syrian, Iraqi, Sudanese and Tunisian. Most cases from Syria were treated in Lebanon, where the number of refugees exceeded 1 million in 2017 (UNHCR, 2017). The Rehabilitation Centre in Tunisia, on the other hand, dealt with torture victims from Tunisia only. Half of the study population was married and lived in households with two to five members. The unemployment rate was relatively high $(46.4 \%)$, while $18.2 \%$ had a full-time job. On average, clients in this study received eight individual NET sessions and the duration of treatment was three months on average with a span from one to eight months. Despite clients' difficulties in remembering when the torture happened, it was possible to obtain data on the time of the most recent torture event for 38 respondents. Most were tortured between 2011 and 2016 (18 respondents), followed by 12 in the 1990s and 7 in the 2000s and a single person in the 1980 s.

Figure 1: Torture methods exposed to (\%)

\section{Torture methods exposed to \%}

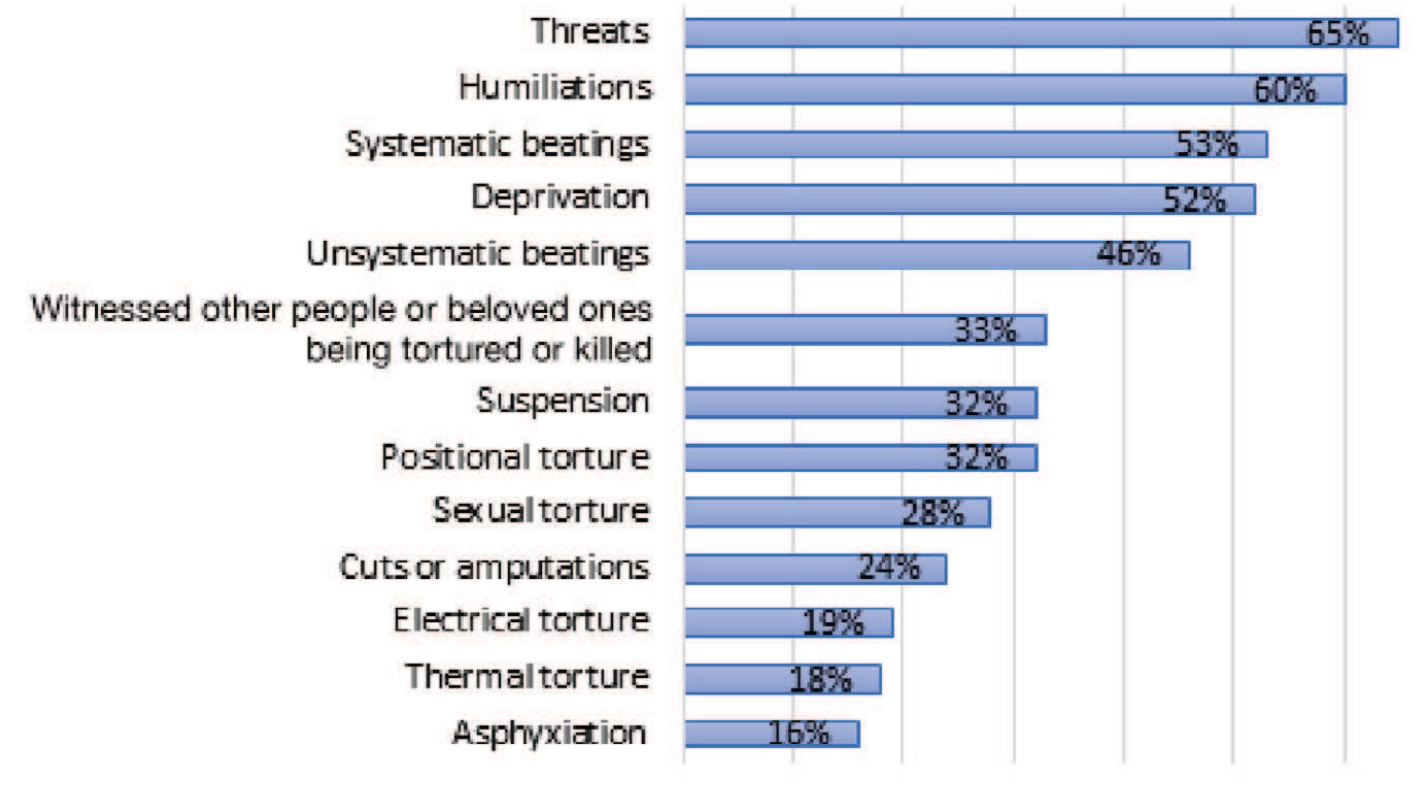


Table 1: Description of the population

\begin{tabular}{ll}
\hline & $\begin{array}{l}\text { Middle east } \\
(\mathrm{n}=110)(\%)\end{array}$ \\
\hline Gender & \\
\hline Male & $63(57.3 \%)$ \\
Female & $38(34.5 \%)$ \\
Missing & $9(8.2 \%)$ \\
\hline
\end{tabular}

Age categories

\begin{tabular}{ll}
\hline $15-17$ & $6(6.6 \%)$ \\
$18-25$ & $5(5.5 \%)$ \\
$26-35$ & $16(14.5 \%)$ \\
$36-45$ & $27(24.5 \%)$ \\
$46-55$ & $18(16.4 \%)$ \\
$56+$ & $10(9.1 \%)$ \\
Missing & $28(25.5 \%)$ \\
\hline
\end{tabular}

Education Level

\begin{tabular}{ll}
\hline None & $3(2.7 \%)$ \\
Primary school & $33(30 \%)$ \\
Secondary school & $24(21.8 \%)$ \\
Vocational education & $14(12.7 \%)$ \\
Higher education & $24(21.8 \%)$ \\
Missing & $12(11 \%)$ \\
\hline
\end{tabular}

\begin{tabular}{ll}
\hline Nationality & \\
\hline Syrian & $37(33.6 \%)$ \\
Iraqi & $15(13.6 \%)$ \\
Sudanese & $12(10.9 \%)$ \\
Tunisian & $25(22.7 \%)$ \\
Libyan & $4(3.6 \%)$ \\
Jordanian & $1(1 \%)$ \\
Palestinian & $10(9.1 \%)$ \\
Egyptian & $1(1 \%)$ \\
Other & $2(1.8 \%)$ \\
Missing & $3(2.7 \%)$
\end{tabular}

Marital status

\begin{tabular}{ll} 
Married & $58(52.7 \%)$ \\
Divorced & $6(5.5 \%)$ \\
Widow(er) & $3(2.7 \%)$ \\
Missing & $10(9.1 \%)$ \\
\hline
\end{tabular}

Employment ${ }^{\star}$

None $\quad 51(46.4 \%)$

Full-time $\quad 20(18.2 \%)$

Self-employed $\quad 14(12.7 \%)$

Part-time $\quad 9(8.2 \%)$

Unpaid housework $\quad 4(3.6 \%)$

Missing $\quad 12(10.9 \%)$

Household members

0-3

$38(41.3 \%)$

4-7

$27(42.3 \%)$

8-11

$15(13.6 \%)$

Missing

$18(16.4 \%)$

* during the last month.

Within this sample, $74.5 \%$ were directly exposed to torture, whereas $13.6 \%$ were traumatised refugees who fled from war. In $11.8 \%$ of the cases, the torture exposure was missing. Methods of torture for this study are shown in Figure 1. Threats, humiliations, beatings and deprivation were the most frequent types of torture.

Direct exposure to beatings of the head can cause loss of consciousness and in severe cases, traumatic brain injury. Further descriptive analysis showed that torture survivors and traumatised refugees were at high risk of brain injury since $54.5 \%$ $(n=60)$ of this sample was exposed to beatings to the head. Among these, $36.4 \%$ $(n=40)$ experienced loss of consciousness. For those exposed to beating to the head, the frequency of beatings was between one and four times. It was important to assess the risk of traumatic brain injury because 
it could limit the benefits from treatment. Relatively more women than men were exposed to sexual torture $(26.8 \%)$ and women $(57.7 \%)$. Pearsons $\chi 2$-test showed statistically significant difference $(p=0.007)$. Sexual torture was often part of other types of torture:

"My family house was attacked. They wanted to take my brother by force-I interferred and shouted, swore at them and attacked them to stop them from taking him. So, they arrested me with him. They covered my head and took me to a house that I don't know. They tied me and started to investigate me, beating me and insulting me-then raped me."

The core analysis of this article examined the effect of NET as changes in psychological symptoms of PTSD, anxiety and depression pre- and post-therapy. Table 2 shows the mean values, number and percentages of clients with a symptom load above the cut-off point for PTSD $(\geq 2.5)$, anxiety and depression $(\geq 1.75)$ pre- and post-treatment. A significant reduction in average psychological symptom load for

Table 2: Pre- and post-assessments of mental health symptoms

\begin{tabular}{|c|c|c|c|}
\hline & $\operatorname{PTSD}(\mathrm{n}=72)$ & Anxiety $(n=71)$ & Depression $(n=67)$ \\
\hline \multicolumn{4}{|l|}{ PRE } \\
\hline Mean pre & 3.2 & 2.78 & 2.75 \\
\hline Above cut-off pre & $91(82.7 \%)$ & $96(87.3 \%)$ & $88(80 \%)$ \\
\hline \multicolumn{4}{|l|}{ POST } \\
\hline Mean post & 1.80 & 1.61 & 1.96 \\
\hline Above cut-off post & $5(4,5 \%)$ & $24(21,8 \%)$ & $60(54.5 \%)$ \\
\hline Mean difference & $1.4 \star$ & $1.17 \star$ & $0.79 \star$ \\
\hline FOLLOW-UP & $\mathrm{n}=12$ & $\mathrm{n}=12$ & $\mathrm{n}=11$ \\
\hline Mean follow-up & 1.58 & 1.61 & 1.72 \\
\hline Above cut-off & 1 & 4 & 4 \\
\hline \multicolumn{4}{|l|}{ Gender comparison } \\
\hline \multicolumn{4}{|l|}{ Male } \\
\hline $\mathrm{n}$ & 40 & 41 & 39 \\
\hline Mean pre & 3.26 & 2.69 & 2.68 \\
\hline Mean post & 1,96 & 1.58 & 2.18 \\
\hline Mean difference & $1.3^{\star}$ & $1.11^{\star}$ & $0.5(p=0.16)$ \\
\hline \multicolumn{4}{|l|}{ Female } \\
\hline $\mathrm{n}$ & 27 & 26 & 25 \\
\hline Mean pre & 3.15 & 2.98 & 2.88 \\
\hline Mean post & 1.65 & 1.7 & 1.7 \\
\hline Mean difference & $1.5 \star$ & 1.28 * & 1.18 * \\
\hline
\end{tabular}

${ }^{\star} \mathrm{p}<0.000$ 
Table 3: Pre- and post-assessment of pyhsical health symptoms

\begin{tabular}{llll}
\hline & Pre & Post & p-value \\
\hline Pain $(\mathrm{n}=61)$ & 4.44 & 3.44 & 0.000 \\
Disability $(\mathrm{n}=57)$ & 2.90 & 1.76 & 0.000
\end{tabular}

PTSD, anxiety and depression was detected. The largest decrease was obtained for PTSD with a decrease of 1.4. The tendency of reduced PTSD and anxiety symptomatology remained the same when adjusting for gender. Gender comparisons revealed higher symptom reductions for females than males. Apart from depressive symptoms for males, all mean differences were highly statistically significant $(\mathrm{p}<0.000)$.

The dropout rate was $23 \%$ since 25 persons had completed the pre-tests, without completion of post-tests. Only full completions of pre-and post-tests were included in the analysis.
In this study, self-rated health was rated significantly $(p=0.004)$ better at the final assessment compared to the first assessment. Before therapy, the mean score was high; 3.85 indicating a poor self-rated health. After therapy, the score declined to 2.82 . We detected a positive shift in all five categories of self-rated health. In the pre-assessment, most cases were distributed in the categories good, fair and poor, whereas in the final assessment, they have shifted to the better categories, including excellent which had not been used in the pre-assessment. At four months' follow-up, data was only obtained from 11 to 12 individuals. The

Figure 2: WHO Disability Assessment Schedule (SUM score 1-60)

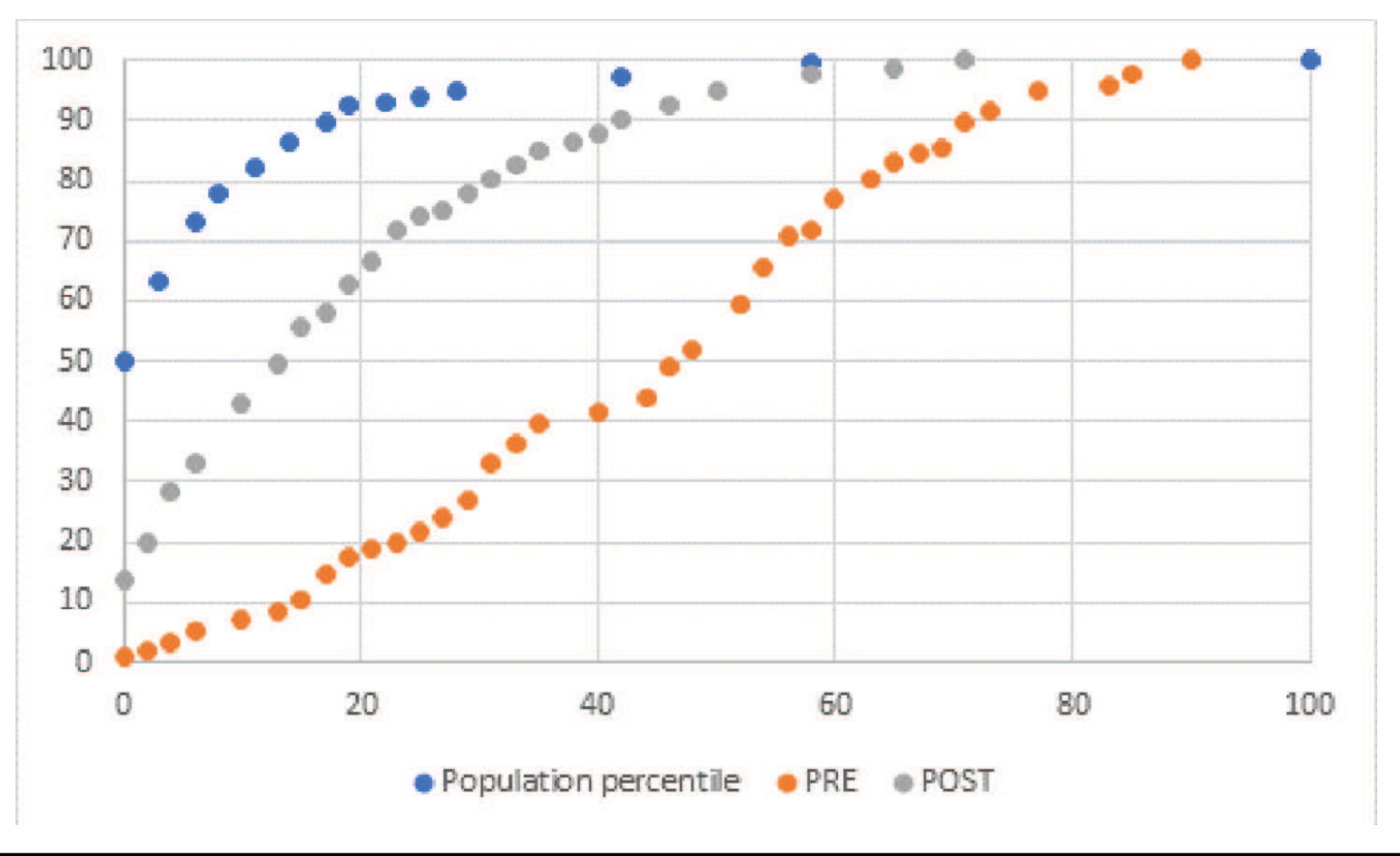


results showed maintained lower levels of PTSD, anxiety and depression, and despite the small sample size, the results were maintained as significant $(p=0.000)$.

The level of pain decreased significantly $(p=0.000)$ with a mean score at 4.44 before NET to 3.44 after NET. The level of disability improved significantly after the last session of therapy. On a scale from 1-5, the mean score was 2.90 before therapy, whereas it decreased to $1.76(p=0,000)$ after therapy. However, comparing the sum-scores in our study to the distribution norms set in the multi-country Survey Study by Üstün TB et al. 2003, both pre- and post-therapy measurements showed extraordinarily higher levels of disability (Figure 2), indicating that torture survivors and traumatised refugees experienced severe functional challenges in their daily lives in addition to mental health problems. The effect of NET reduced the symptoms of disability, but our sample is still half of the normal distribution.

In terms of clients' self-reported experiences of attending NET, the qualitative evaluations showed torture survivors and traumatised refugees felt their relations with others and communication had improved, as exemplified here:

"The therapy made me a social person. My self-confidence is better, I learned to speak about what is hurting me to the specialist. I feel more relaxed - I was afraid of people after what happened to me but thank God I am dealing with everyone in a better way and I am not scared anymore."

Our study participants expressed better integration into society, e.g., as increased participation; felt they could have a role in their lives; experienced less fear; and, finally, showed improved sleeping with fewer nightmares:

"When speaking about memories and hurting events the psychological effect is less, dreams and nightmares are less, especially those connected to the shocking events. There is still a percentage of anxiety and depression due to reasons of 'no income' and 'worries about the future of my children' and then I am also scared that my husband will leave my children and run away from his duties and expenses."

The decreased prevalence of nightmares was substantiated by an analysis of a single item in the HTQ, asking about recurrence of nightmares. Our results show that nightmares on average decreased significantly from 3.03 to $1.44(p=0,005)$ on a score from 1-4.

\section{Discussion}

NET improves psychosomatic health in the MENA region: Our findings show a strong statistically significant reduction in symptoms of PTSD, anxiety and depression amongst survivors of torture who received NET in the MENA region in the period 2013-2016. NET had its greatest impact on PTSD symptoms and its lowest on depression symptoms. This result is not surprising since NET targets PTSD and not depression. So even though we know that the symptoms are interlinked, we did not expect a high impact on depression. One theory of explanation is that traumatised individuals who come from and live in shame-based societies such as those in the MENA region (Fessler, 2004) may be impacted by their upbringing and culture where one's inability to fulfill obligations and live up to others' expectations is considered to be monumental. Specifically, it is possible that trauma victims who are experiencing a low level of functioning and unable to perform their social roles might see themselves as being in an inferior position, and subsequently adopt non-assertive submissive and submissive defensive behaviors. These 
behaviours and emotions have been linked to depression (Gilbert, 2016).

This study assesses the effect of NET on anxiety. Likewise, this study is the first to present somatic effects of NET, showing improvements in self-reported health as well as a reduction in pain perceptions and levels of disabilities after treatment. This points to a positive association between psychological and somatic health outcomes related to NET. Furthermore, our findings show a larger effect for women than men. This supports previous literature that women benefit more from trauma therapy than men (Békés, Beaulieu-Prévost, Guay, Belleville, \& Marchand, 2016). In the present study, it is possible that men found the NET requirement to relive the traumatic experience and express feelings and associated physiological responses as more challenging. The exposure element of NET may be particularly difficult for men in male-dominated societies such as in the MENA region. So far, the effects of NET on PTSD and depression in torture survivors have mainly been evaluated in high-income settings. This research evaluates the effect of NET on PTSD and depression in torture victims who continue to live in unsafe and difficult environments facing legal, employment, and financial challenges in their current middle- or low income countries. Some faced threats of deportation back to their home country on a regular basis, and some were still at risk of being tortured. This research rejects the assumption that trauma treatment should be delayed until refugees or asylum seekers resettle to live in a stable and permanent environment (Norredam, Mygind, \& Krasnik, 2006). Instead, the current findings coincide with previous studies suggesting treatment need not be delayed (Stenmark et al., 2013).
Do daily stressors affect the effect of NET on mental health? Our research indicates that daily stressors affect the effect of NET on PTSD symptoms, supporting previous evidence (Miller and Rasmussen, 2010). Other important factors besides the NET treatment model may explain why our participants from the MENA region experienced more significantly reduced PTSD symptoms than those from highincome countries. One might be the context surrounding the client while receiving therapy, which in our study population was familiar cultural norm settings. Another aspect might be whether the therapist shares the same cultural background as well as language with the client, since socio-cultural knowledge and cultural competency of the therapist are crucial in the collaboration with the client (Hassan et al., 2016). In the case of our study population the NET therapists had extensive cultural competencies, for some due to shared nationality between therapist and client, and no interpreters were necessary in the therapeutic sessions. Finally, the client's social status and feeling of identity may play an important role in the effect of therapy, which is often related to asylum or refugee status. In our study population about $50 \%$ were employed, where, in contrast, the employment rate may be much lower among refugees living in camps. Hassan et al. (2016) suggests that improved living conditions and livelihoods may improve the mental health of refugees and internally displaced persons even more than psychological and psychiatric interventions.

Strengths and limitations: Despite some important limitations, this study represents new evidence on the effect of NET among torture survivors in the MENA region. Limitations include that it was carried 
out without a control group, which made it impossible to rule out spontaneous improvement, or improvement due to other treatments, medication, and/or because of environmental, psychosocial and community changes. This is particularly the case as the measures used were mostly related to symptoms and not to other aspects related to the deep experience of survivors. This is something that could be clarified with further studies including a control group (for instance through having similar measures with waiting lists of participants).

The risk of information bias should be considered as the therapist collected data prior to, and after therapy, which can cause an overestimation of the effect of the therapy. On the other hand, it is likely that self-reported psychological symptoms were underestimated because of stigmatisation of mental health challenges in the Middle East. That said, reliability of the reported outcomes was strengthened since reporting a positive progress of their therapy outcomes was potentially against their self-interest; a significant proportion of treated clients had applied for refugee status and severe mental health symptoms can be seen as having an advantage over others in terms of being granted refugee status.

This study had a relatively high dropout rate, but this should be seen in light of the difficult contexts the clients lived in. Most clients lived illegally in their hosting countries; Jordan, Lebanon and Egypt. They faced daily threats of immediate deportation by authorities, or physical harassment or abuse by local nationals. Two main reasons for dropout of treatment were that some felt the need to move their housing location when they felt threatened. Secondly male clients, who had to work illegally to support their families suddenly moved once they found a new job in a different place without giving prior notice to the therapists.

Despite the intention of collecting follow-up data after four months, the response rate was very low (12 clients) since many clients were refugees and had migrated after four months. The data is thus insufficient to estimate the long-term effects of NET. Finally, there was a large amount of missing data, which can cause biased estimates and decreased statistical power, as well as weakened generalisability. In the core analysis of this study, we only included cases where clients had completed full pre-and post- scales for PTSD, anxiety and depression. As all findings were highly significant, it seems likely that the results could be generalised to a larger sample with high compliance to the treatment. However, the missing data may have underor overestimated the detected larger effect of NET among women.

Strengths include that the multicentre approach enabled us to test the applicability of NET on a large number of NET cases across the MENA region since many therapists from different countries performed this therapy in their respective country contexts. The significant results dispersed over a large group of therapists also strengthened the reliability of the results. The application of mixed methods with openended answering options in connection to the quantitative measures increased the validity of the research. Furthermore, all therapists received monitoring and evaluation training prior to using the tool, increasing both validity and reliability.

Implications for practice: Our results can inform decisions in clinical practice and in policy-making, suggesting a positive influence of familiar contexts and cultural norm settings surrounding clients while 
receiving NET, as well as highlighting the importance of the therapist having a high level of cultural competency, and competency in the same language as the client with no need to use interpreters during sessions.

In conclusion, this educational project managed to fill a gap in the mental health arena in the MENA region by establishing a community of Arab mental health specialists within trauma counselling in the region with promising results that strongly suggest that short-term NET therapy can significantly reduce the mental health symptom load of survivors of war and torture.

\section{Acknowledgements}

We would like to thank all the health professionals of the participating rehabilitation centres in the MENA region for their support and collaboration in the data collection process. Importantly, we are also grateful to the NET clients for the invaluable information provided for this study.

\section{References}

Adenauer, H., Catani, C., Aichinger, H., Keil, J., Ruf, M., \& Neuner, F. (2011). Narrative Exposure Therapy for PTSD increases top-down processing of aversive stimuli - evidence from a randomized controlled treatment trial. $B M C$ Neuroscience, 12, 127.

Békés, V., Beaulieu-Prévost, D., Guay, S., Belleville, G., \& Marchand, A. (2016). Women with PTSD benefit more from psychotherapy than men. Psychol Trauma, 8, 720-727.

Bernard, J. M., \& Goodyear, R. K. (2009). Fundamentals of clinical supervision ( $4^{\text {th }}$ ed.). Needham Heights, MA: Allyn \& Bacon.

Brewin, C. R., \& Holmes, E. A. (2003). Psychological theories of posttraumatic stress disorder. CLIN PSYCHOL REV, 23, 339 - 376.

Budosan, B., Aziz, S., Benner, M. T., \& Abras, B. (2016). Perceived needs and daily stressors in an urban refugee setting: Humanitarian Emergency Settings Percieved Needs Scale of Syrian refugees in Kilis, Turkey. Intervention 1-12.
Crumlish, N., \& O'Rourke, K. (2010). A systematic review of treatments for post-traumatic stress disorder among refugees and asylum-seekers. f Nerv Ment Dis., 198, 237-51. doi: 10.1097/ NMD.0b013e3181d61258.

Fessler, D. (2004). Shame in Two Cultures: Implications for Evolutionary Approaches. Fournal of Cognition and Culture 4.2

Gilbert, P. (2016). Depression: The Evolution of Powerlessness. Rouledge: NY.

Gwozdziewycz, N., \& Mehl-Madrona, L. (2013). Meta-Analysis of the Use of Narrative Exposure Therapy for the Effects of Trauma Among Refugee Populations. The Permanente fournal 17. Doi: http://dx.doi.org/10.7812/TPP/12-058.

Hassan, G., Ventevogel, P., Jefee-Bahloul, H., \& Barkil-Oteo, A. (2016). Mental health and psychosocial wellbeing of Syrians affected by armed conflict. Epidemiology and Psychiatric Sciences, 25, 129-141.

Hensel-Dittmann, D., Schauer, M., Ruf, M., Catani, C., Odenwald, M., Elbert, T., \& Neuner, F. (2011). Treatment of Traumatized Victims of War and Torture: A Randomized Controlled Comparison of Narrative Exposure Therapy and Stress Inoculation Training. Psychotherapy and Psychosomatics, 80, 345-352.

Hijazi, A. M., Lumley, M. A., Ziadni, M. S., Haddad, L., Rapport, L.J., \& Arnetz, B.B. (2014). Brief narrative exposure therapy for posttraumatic stress in Iraqi refugees: a preliminary randomized clinical trial. F Trauma Stress, 27, 314-22. doi: $10.1002 /$ jts. 21922 .

Idler, E. L. \& Benyamini, Y. (1997). Self-Rated Health and Mortality: A Review of Twenty-Seven Community Studies. Fournal of Health and Social Behavior, 38, 21-37.

Konecky B, Meyer EC, Marx BP, et al (2014). Using the WHODAS 2.0 to assess functional disability associated with DSM-5 mental disorders. American fournal of Psychiatry 171, 818-820.

Mahfoud, Z., Kobeissi, L., Peters, T. J., Araya, R., Ghantous, Z., \& Khoury, B. (2013). The Arabic Validation of the Hopkins Symptoms Checklist-25 against MINI in a Disadvantaged Suburb of Beirut, Lebanon. International fournal of Educational and Psychological Assessment, 13, 17-33.

Miller, E. K., Rasmussen, A. (2010). War Exposure, Daily Stressors, and Mental Health in Conflict and Post-Conflict Settings: Bridging the Divide between trauma-focused and psychosocial frameworks. Social Science and Medicine, 70, 7-16.

Mollica, R.F., Caspi-Yavin, Y., Bollini, P., Truong, T., Tor, S., Lavelle, J. (1992). The Harvard Trauma Questionnaire. Validating a cross-cultural 
instrument for measuring torture, trauma, and posttraumatic stress disorder in Indochinese refugees. F Nerv Ment Dis. 180, 111-6.

Morath, J., Gola, H., Sommershof, A.., Hamuni, G., Kolassa, S., Catani, C., Adenauer, H., Ruf-Leuschner, M., Schauer, M., Elbert, T., \& Groettrup, M. (2014). The effect of trauma-focused therapy on the altered $\mathrm{T}$ cell distribution in individuals with PTSD: Evidence from a randomized controlled trial. fournal of Psychiatric Research 54: 1-10. doi: 10.1016/j. jpsychires.2014.03.016.

Mørkved, N., Hartmann, K., Aarsheim, L. M., Holen, D., Milde, A. M., Bomyea, J., \& Thorp, S. R. (2014). A Comparison of Narrative Exposure Therapy and Prolonged Exposure Therapy for PTSD. Clinical Psychology Review, 34, 453-467. doi: 10.1016/j.cpr.2014.06.005.

Neuner, F., Kurreck, S., Ruf, M., Odenwald, M., Elbert, T., \& Schauer, M. (2010). Can AsylumSeekers with Posttraumatic Stress Disorder Be Successfully Treated? A Randomized Controlled Pilot Study. Cognitive Behaviour Therapy, 39, 81-91.

Neuner, F., Onyut, P.L., Ertl, V., Odenwald, M., Schauer, E., \& Elbert T. (2008). Treatment of posttraumatic stress disorder by trained lay counselors in an African refugee settlement: a randomized controlled trial. $\mathcal{F}$ Consult Clin Psychol 76,686-94. doi: http://dx.doi.org/10.1037/0022006X.76.4.686.

Neuner, F., Schauer, M., Klaschik, C., Karunakara, U., \& Elbert, T. (2004). A comparison of narrative exposure therapy, supportive counseling, and psychoeducation for treating posttraumatic stress disorder in an African refugee settlement. $\mathcal{F}$ Consult Clin Psychol 72,579-87. doi: http://dx.doi. org/10.1037/0022006X.72.4.5792.

Norredam, M., Mygind, A., Krasnik, A. (2006). Access to health care for asylum seekers in the European Union - a comparative study of country policies. Eur F Public Health, 16, 285-289. doi: https://doi.org/10.1093/eurpub/cki191.

Nosè, M., Ballette, F., Bighelli, I., Turrini, G., Purgato, M., Tol, W., Priebe, P., \& Barbui, C. (2017). Psychosocial interventions for posttraumatic stress disorder in refugees and asylum seekers resettled in high-income countries: Systematic review and meta-analysis. PLoS ONE, 12, e0171030. doi: 10.1371/journal. pone.0171030.

Robjant, K., \& Fazel, M. (2010). The Emerging Evidence for Narrative Exposure Therapy. Clinical Psychology Review, 30, 1030-39.
Schaal, S., Elbert, T., \& Neuner, F. (2009). Narrative exposure therapy versus interpersonal psychotherapy. A pilot randomized controlled trial with Rwandan genocide orphans. Psychotherapy and Psychosomatics, 78, 298-306.

Schauer, M., Neuner, F., \& Elbert, T. (2005). Narrative Exposure Therapy - a short term intervention for traumatic stress disorders after war, terror or torture. Cambridge, MA: Hogrefe \& Huber.

Schnittker, J., \& Bacak, V. (2014). The Increasing Predictive Validity of Self-Rated Health. PLoS ONE 9. https://doi.org/10.1371/journal. pone.0084933.

Shoeb, M., Weinstein, H., \& Mollica, R. (2007). The Harvard Trauma Questionnaire: Adapting a Cross-Cultural Instrument for Measuring Torture, Trauma and Posttraumatic Stress Disorder in Iraqi Refugees. International fournal of Social Psychiatry.

Stenmark, H., Catani, C., Neuner, F., Elbert, T., \& Holen, A. (2013). Treating PTSD in refugees and asylum seekers within the general health care system. A randomized controlled multicenter study. Behav. Res. Ther., 1, 641-7.

UNHCR (2017). Populations. Retrieved from URL: http://reporting.unhcr.org/population.

Üstün TB et al. (2003). WHO multi-country survey study on health and responsiveness 2000-2001. In: Health systems performance assessment: debates, methods and empiricism. Geneva, World Health Organization, 2003, 761-796.

WHO (2010) Measuring Health and Disability: Manual for WHO Disability Assessment Schedule (WHODAS 2.0)/ Üstün, T B., Kostanjsek, N., Chatterji, S., \& Rehm, J. (Eds)

Whaley, A. L., \& Davis, K. E. (2007). Cultural Competence and Evidence-Based Practice in Mental Health Services: A Complementary Perspective. American Psychologist, 62, 563-574. doi: 10.1037/0003-066X.62.6.563.

WHO (2014). Global Health Observatory data repository. Retrieved from URL: http://apps.who. int/gho/data/node.main.MHHR?lang=en.

Zaharna, R. (1995). Bridging Cultural Differences: American Public Relations Practices \& Arab Communication Patterns. Public Relations Review, 21, 241-255. 